\title{
Severe Chronic Neck Injury Caused by a Snare in a Coyote, Canis latrans
}

\author{
PierRe-Yves DaOUST ${ }^{1,3}$ and Peter H. Nicholson ${ }^{1,2}$ \\ ${ }^{1}$ Canadian Cooperative Wildlife Health Centre, Department of Pathology and Microbiology (Daoust), and Class of 2002 \\ (Nicholson), Atlantic Veterinary College, University of Prince Edward Island, 550 University Avenue, Charlottetown, \\ Prince Edward Island CIA 4P3, Canada \\ ${ }^{2}$ Present address: College Village Animal Clinic, 2036 E. Northern Lights Boulevard, Anchorage, Alaska, 99508, USA \\ ${ }^{3}$ Corresponding author: e-mail: daoust@upei.ca
}

Daoust, Pierre-Yves, and Peter H. Nicholson. 2004. Severe chronic neck injury caused by a snare in a Coyote, Canis latrans. Canadian Field-Naturalist 118(2): 243-246.

A two-year-old male Coyote, Canis latrans, in poor body condition was found in a moribund state with a snare deeply embedded in the ventral portion of its neck, more than a month after the official end of the trapping season on Prince Edward Island. This snare had presumably malfunctioned, and the cable had cut through the soft tissues of the neck as well as the trachea and had obstructed both jugular veins and both common carotid arteries but had largely spared both vagosympathetic trunks. Cases like this illustrate the need to continue to work on improving the efficiency of trapping methods, through research and trapper education.

Key Words: Coyote, Canis latrans, trapping, injury, snare, Prince Edward Island.

Animal welfare issues surrounding trapping methods used to capture furbearers have been an ongoing topic of discussion between wildlife managers and animal protection groups for many years (Proulx and Barrett 1991). Because of this and of the economic importance of the fur trade to Canada, this country has taken a leading role in developing national and international standards for the performance of restraining- and killing-type traps. In the section of the Agreement on International Humane Trapping Standards (AIHTS) $(1997 *)$ dealing with killing type traps such as the commonly used rotating-jaw Conibear trap, the designated time limit to irreversible loss of corneal reflexes in at least $80 \%$ of the animals of the target species caught in the trap varies from 45 seconds in Ermine (Mustela erminea) to 120 seconds in Pine Marten (Martes americana) to 300 seconds in 17 other species of North American and European furbearing animals. This Agreement requires that the parties involved continue research with a view to lowering the threshold requirements. Nonetheless, it implicitly recognizes that no killing trapping method used to capture wild animals can as yet guarantee a humanely acceptable process in each instance. Manual neck snares (where the animal provides the energy necessary to tighten the snare) are widely used by trappers to capture various furbearing animals, and Guthery and Beasom (1978) and Boddicker (1982) discussed the advantages and disadvantages of snares as compared to other trapping devices. Snares are allowed in most Canadian jurisdictions, except the southern regions of some provinces. Being considered devices that are constructed by the trappers themselves, they are not subject to the testing requirements under the AIHTS (1997), but their design must be approved by relevant competent authorities such as provincial Departments of Wildlife. This article describes an unsuccessful capture by snare of a Coyote (Canis latrans), with severe consequences.

\section{Case Description}

On 26 February, 2002, a male Coyote, subsequently determined to be 2 years old (Matson's Laboratory, Milltown, Montana, USA), was found alive but moribund in a field in Kings County $\left(46^{\circ} 08^{\prime} \mathrm{N}, 62^{\circ} 36^{\prime} \mathrm{W}\right)$, Prince Edward Island (PEI), and was euthanized by a shot to the head. It was submitted for necropsy to the Diagnostic Laboratory of the Atlantic Veterinary College, University of PEI. Postmortem examination revealed that the animal had been caught in a snare and had managed to escape. The snare's cable, made of galvanized steel wire $2 \mathrm{~mm}$ in diameter, was equipped with a standard U-shaped lock, similar to an Adams lock. The snare surrounded the animal's neck, with a portion of cable approximately $20 \mathrm{~cm}$ long dangling beyond the neck. The coyote was in poor body condition; the stomach was completely empty, and the intestines contained only a small amount of ingesta. However, some fat was still present around the base of the heart, and a sample of diaphyseal bone marrow from one of the femurs contained approximately 54\% fat, as measured by comparison of the wet and dry weights of the sample. A large area of the skin along the ventral side of the neck, about $50 \mathrm{~cm}^{2}$, was missing and had been replaced by scar tissue that released a foul odour indicative of suppuration. The portion of the cable along the ventral side of the neck had transected the full diameter of the trachea and was embedded in scar tissue between the trachea and the esophagus (Figure 1). The wall of the severed portion of the trachea was completely healed, although the tissue of repair had extended as a thin circular band 
into the tracheal lumen, reducing its surface area to about one-seventh of its original size (Figure 2). The esophageal lumen was patent. There was no gross evidence of suppuration accompanying the scar tissue that surrounded the trachea and the cable. At the time of necropsy, the cable was freely movable within the affected tissues. The architecture of both jugular veins disappeared at the level of the scar tissue. A thin probe inserted into the cranial portion of the common carotid artery on both sides of the trachea was blocked from further progression at this level. The outline of the vagosympathetic trunk on both sides was also lost among the scar tissue. No other significant lesion was seen at necropsy. Samples of the affected portion of trachea and surrounding soft tissues were fixed in formalin, processed routinely for microscopic examination, and stained with hematoxylin and eosin.

Microscopically, the scar tissue surrounding the affected portion of trachea consisted of abundant dense fibrous tissue, much of which was birefringent under polarized light. This fibrous tissue enclosed several pockets of necrotic material (including debris of inflammatory cells) and, in some areas, contained numerous cross sections of hair shafts. Serial sections of this tissue, parallel to the length of the trachea, revealed on both sides an artery, about $3 \mathrm{~mm}$ in diameter, and a nerve, about $1 \mathrm{~mm}$ in diameter. Taking into account the shrinkage that normally occurs in tissues processed for microscopic examination, the diameters of these two structures were compatible with those of the common carotid artery and vagosympathetic trunk, respectively, for an animal of this size. Depending on the section examined, the lumen of the common carotid artery on both sides of the trachea was either empty or partly or completely filled with a well organized thrombus. The vagosympathetic trunk on both sides was morphologically normal, except for a few degenerative lesions suggesting some loss of axons and their myelin sheaths (wallerian degeneration). The tissue within the tracheal lumen also consisted of dense fibrous tissue that enclosed some necrotic material and numerous cross sections of hair shafts.

\section{Discussion}

Ideally, a snare should cause rapid death by neck strangulation and subsequent asphyxiation. A quick death is more humane and also gives the animal less time to find a way out of the snare. A variety of factors can influence the performance of a snare. Some locking mechanisms tighten less quickly and firmly than others (Phillips 1996), or small kinks in the cable may weaken it and make it easier to break or may prevent full function of the lock. Inclusion of a swivel attaching the lock to the cable (not present in this case) can prevent the cable from kinking as the lock slides along it. If the cable is not anchored firmly enough, the animal may be able to pull off the snare before maximum closure has been achieved. If death is not rapid and the cable is anchored too low, it may be more easily accessible to the animal's jaws and be chewed through. Seventeen of 131 (13\%) Coyotes caught by one of three types of snares studied by Phillips (1996) managed to escape, one by breaking the lock and 16 by chewing through the cable.

In this Coyote, the relative abundance and density of the scar tissue in the neck and, microscopically, its birefringence under polarized light suggested that the failed capture had occurred at least a few weeks prior to death. The trapping season for Coyote and Red Fox (Vulpes vulpes) on PEI officially terminated on January 15 , more than a month before this animal was found. The reason why it managed to escape capture was not determined, although the portion of cable dangling from its neck was of the right length to suggest that the animal had chewed through it rather than pull the whole cable from where it was anchored. Unfortunately, the animal's gums and teeth were not examined for the presence of recent damage that would have supported this suggestion. At the time of escape, sufficient closure of the snare may have already been achieved for the cable to exert a strong pressure on soft tissues of the ventral side of the neck and be forced gradually through these tissues over the following weeks. Gradual transection and concurrent repair of the tracheal wall would thus have allowed it to remain continuous. Alternatively, transection of all soft tissues, including the trachea, could have occurred at the time of the animal's initial struggle in the snare. Such rapid transection of the trachea, however, would more likely have resulted in the formation of a gap between its cut ends, thus preventing them from healing together. Moreover, if the trachea had been rapidly transected, the jugular veins would probably also have been cut and the animal would presumably have bled to death. The thicker hair coat and skin on the dorsal side of the neck as compared to the ventral side (Scott et al. 2001) and the tough nuchal ligament on the dorsal side (Evans 1993) may have prevented the cable from moving into the tissues from that side.

The common carotid arteries and the vagus nerves are important structures running along both sides of the trachea. In this animal, the lumen of both common carotid arteries was occluded. However, this is not a critical problem, at least in canids. In dogs, both common carotid arteries can be ligated experimentally without causing any clinical signs, implying that collateral circulation (e.g., via the vertebral arteries) is able to quickly compensate and transport sufficient blood to the brain (Whisnant et al. 1956). Such collateral circulation would have even more time to develop if the obstruction were gradual, as is suspected to have occurred in this case. The same reasoning applies to the jugular veins, located much more superficially in the ventral region of the neck, since collateral circulation within the venous system can develop even more readily than in the arterial side. Proper function 


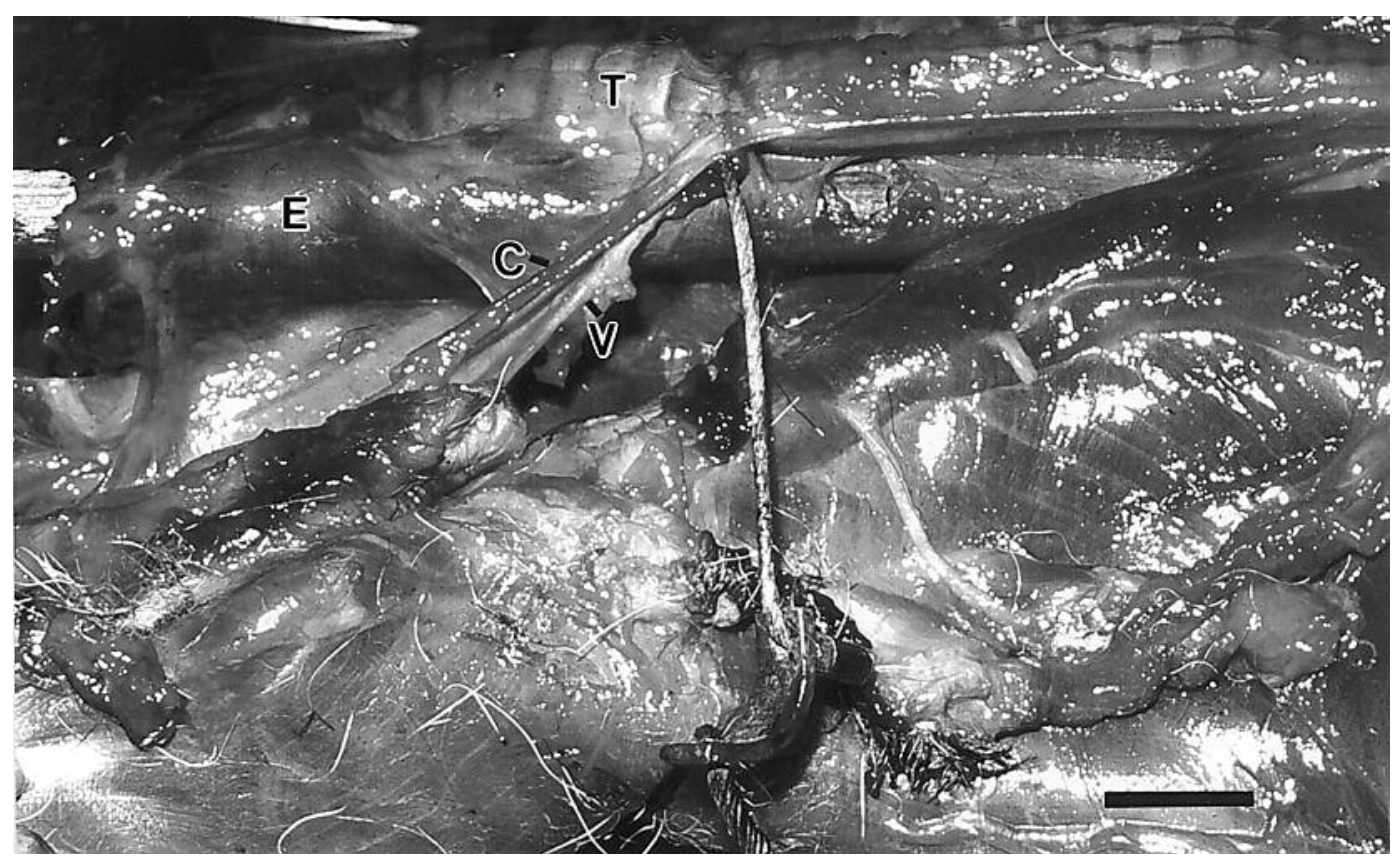

FIGURE 1. Right lateral view of the neck of a Coyote. The carcass is lying on its back, with the head to the left. The cable from a snare has cut through the trachea $(\mathrm{T})$ and is now lodged between it and the esophagus (E). The distortion of the wall of the trachea just above the cable as been caused by scar tissue of repair. A metal rod has been inserted in the lumen of the esophagus. The common carotid artery $(\mathrm{C})$ and vagosympathetic trunk $(\mathrm{V})$ are clearly visible to the left of the cable. The lock of the snare is at the bottom of the picture. (Bar $=2 \mathrm{~cm}$.)

of at least one of the vagosympathetic trunks is, however, essential to life. The vagal (parasympathetic) portion of these nerves originates in the brain and supplies efferent and afferent innervation to many vital organs, including the cardiovascular system and digestive tract (Andrews and Lawes 1992; Esler 1992; Guyton and Hall 1996). In particular, vagal stimulation protects against excessive stimulation of cardiac function by the sympathetic nervous system during physical activity and stress. In this Coyote, it was not possible to dissect the vagosympathetic trunk on either side of the affected portion of trachea, because both trunks were well embedded in scar tissue at this level. However, careful microscopic examination showed that both trunks were in large part morphologically intact. Although their structure had likely been stretched, and their path distorded, by the cable, the gradual process through which this happened would presumably have provided sufficient time for anatomic and physiological compensation.

The exact cause of the eventual demise of this coyote was not clear. There are undocumented reports by veterinary practitioners of collars being left permanently on growing dogs and eventually becoming buried under the skin, with damage limited to chronic suppuration of the subcutis (J. B. Miller, Department of Companion Animals, Atlantic Veterinary College, University of PEI, personal communication). Similarly, a square of a monofilament gillnet mesh was found almost totally embedded in the snout of an otherwise healthy Porbeagle (Lamna nasus) that had been caught on the Scotian Shelf (Benz et al. 2001). However, the marked reduction in size of the tracheal lumen in this Coyote may have reduced its stamina and prevented it from foraging efficiently. The esophageal lumen was

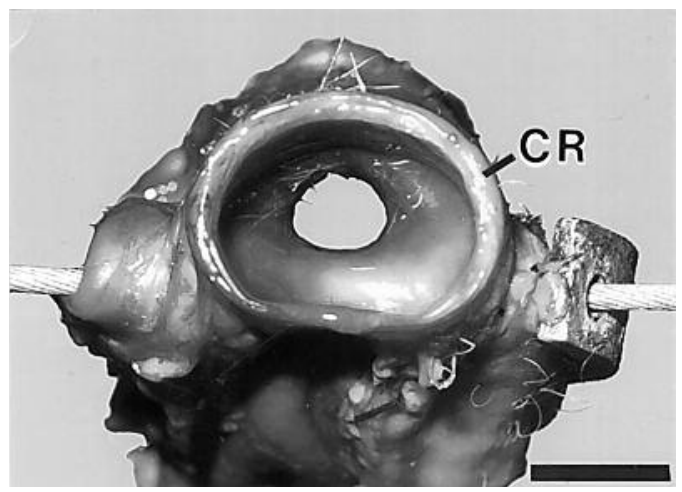

FIGURE 2. Cranial view of the trachea of a Coyote at the level where the cable of a snare has cut through this organ. The ventral side of the trachea is at the top. The cartilaginous ring $(\mathrm{CR})$ outlines what should be the normal size of the tracheal lumen. Scar tissue of repair has greatly narrowed this lumen. $(\mathrm{Bar}=1 \mathrm{~cm}$.) 
patent, but the presence of the cable against its ventral surface may have interfered with the passage of large food boluses. Albeit seemingly superficial, the suppurative process and, therefore, probable bacterial infection, associated with the soft tissue damage would have further drained the animal's energy reserves. Although this animal had not yet reached a stage of emaciation, its poor body condition, combined with the energy demands imposed by the winter season, may have been sufficient to bring it to a stage of exhaustion, perhaps combined with hypothermia. The clinical significance, if any, of the mild damage seen microscopically in the vagosympathetic trunks could have been assessed only in the live animal.

The efficacy of various trapping devices, including snares, can now be properly tested under standardized laboratory conditions (Proulx and Barrett 1990, 1991). Ultimately, however, the proportion of animals that fail to die rapidly and undergo prolonged suffering depends much on the experience and expertise of the trappers in the field. Regardless of the exact cause of failure of the snare in this case, its outcome emphasizes the need for continued attention to appropriate training of hunters and trappers, in order to ensure that the best trapping devices and capture techniques are used consistently and, as a result, as few animals as possible undergo undue suffering. It also illustrates the extreme resilience of Coyotes.

\section{Acknowledgments}

This article benefited greatly from discussions with Neal Jotham.

\section{Documents Cited [marked * in text]}

Agreement on International Humane Trapping Standards. The European Community, the Government of Canada, and the Government of the Russian Federation. 1997. Department of Foreign Affairs and International Trade, Ottawa, Ontario, Canada. 31 pages.

\section{Literature Cited}

Andrews, P. L. R, and I. N. C. Lawes. 1992. A protective role for vagal afferents: an hypothesis. Pages 280-302 in Neuroanatomy and physiology of abdominal vagal afferents. Edited by S. Ritter, R. C. Ritter and C. D. Barnes. CRC Press, Boca Raton, Florida, 316 pages.

Benz, G. W., A. Kingman, and J. D. Borucinska. 2001. Gillnet survival and healing by a Porbeagle, Lamna nasus. Canadian Field-Naturalist 115: 506-509.

Boddicker, M. L. 1982. Snares for predator control. Proceedings of the Vertebrate Pest Conference 10: 50-54.

Esler, M. 1992. The autonomic nervous system and cardiac arrhythmias. Clinical Autonomic Research 2: 133-135.

Evans, H. E. 1993. Arthrology. Pages 219-257 in Miller's anatomy of the dog. Edited by H. E. Evans. W. B. Saunders Company, Toronto, $3^{\text {rd }}$ edition, 1113 pages.

Guthery, F. S., and S. L. Beasom. 1978. Effectiveness and selectivity of neck snares in predator control. Journal of Wildlife Management 42: 457-459.

Guyton, A. C., and J. E. Hall 1996. Textbook of medical physiology. W. B. Saunders Company, Toronto, $9^{\text {th }}$ edition: pages 107-119, 793-802.

Phillips, R. L. 1996. Evaluation of 3 types of snares for capturing coyotes. Wildlife Society Bulletin 24: 107-110.

Proulx, G., and M. W. Barrett. 1990. Assessment of power snares to effectively kill red fox. Wildlife Society Bulletin 18: 27-30.

Proulx, G., and M. W. Barrett. 1991. Ideological conflict between animal rightists and wildlife professionals over trapping wild furbearers. Transactions of the North American Wildlife and Natural Resources Conference 56: 387-399.

Scott, D. W., W. H. Miller Jr., and C. E. Griffin. 2001. Muller \& Kirk's small animal dermatology. W. B. Saunders Company, Toronto, $6^{\text {th }}$ edition, pages 1-70.

Whisnant, J. P., C. H. Millikan, K. G. Wakim, and G. P. Sayre. 1956. Collateral circulation to the brain of the dog following bilateral ligation of the carotid and vertebral arteries. American Journal of Physiology 186: 275-277.

Received 7 February 2003

Accepted 26 October 2004 